\title{
TABLES FOR TESTING THE GOODNESS OF FIT OF THEORY TO OBSERVATION.
}

\author{
By W. PALIN ELDERTON, Aetuary.
}

[Received October 18, 1901.]

On the Test for Random Sampling.

ANY theoretical description by means of curve or series is ceteris paribus admissible as a graduation of a given set of frequency observations, provided the observed values do not differ from the values provided by this theory by more than the reasonable deviations due to random sampling. There may be utilitarian reasons (eg. relative fewness of descriptive constants, or their easy calculation) or philosophical reasons (e.g. general theories as to the nature and distribution of causes producing frequency phenomena) why we should adopt one theoretical description rather than nnother, but apart from such reasons that theoretical description is best, which describes the observed frequencies with the "greatest probability." By "describing the observed frequencies with the greatest probability" we understand a good although conventional test of fitness. Suppose the theoretical description of the frequencies to be the actual distribution of the whole population; we ask in how many cases per 100 in a series of random samplings should we differ from the theoretical distribution by as wide a system of deviations as that observed, or by a still wider system? In other words we want to find out the probability $P$ that in random sampling deviation-systems as great as or greater than that actually observed will arise. This point has beeu dealt with in a paper by Professor K Pearson published in the Philosophical Magasine*, and it is there shown that if there be $n^{\prime}=n+1$ frequency groups in the series, and $m_{r}$ and $m_{r}^{\prime}$ be the theoretical and observed frequencies in any group, it is necessary to find

$$
\chi^{2}=S\left\{\frac{\left(m_{r}-m_{r}\right)^{2}}{m_{r}}\right\}=\operatorname{sum}\left(\frac{\left\{\begin{array}{c}
\text { squares of differences of theoretical } \\
\text { and observed frequencies }
\end{array}\right\}}{\text { theoretical frequency }}\right),
$$

- On the Criterion that a given 8ystem of Deviations from the Probable in the case of a Correlated Byatem of Variables is sabh that it an be reasonably mpposed to heve arien trom Random Bampling, Vol. I. Pp. 157-176, July, 1800. 
and that $P$ will then be calculated from:

$$
P=\sqrt{\frac{\overline{2}}{\pi}} \int_{x}^{\infty} e^{-k x^{2}} d x+\sqrt{\frac{2}{\pi}} e^{-t x^{0}}\left(\frac{\chi}{1}+\frac{\chi^{2}}{1.3}+\frac{\chi^{2}}{1.8 .5}+\ldots+\frac{x^{n^{\prime}-}}{1.3 .5 \ldots\left(n^{\prime}-3\right)}\right)
$$

if $n^{\prime}$ be even, and from:

if $n^{\prime}$ be odd.

$$
P=0^{-t x^{2}}\left(1+\frac{\chi^{2}}{2}+\frac{\chi^{x}}{2.4}+\frac{x}{2.4 .6}+\ldots+\frac{\chi^{n^{\prime}-8}}{2.4 .6 \ldots\left(n^{\prime}-8\right)}\right)
$$

Now although $\chi^{2}$ can be found quite easily without any special mathematical knowledge, the calculation of $P$ from the sbove formula is very troublesome. But it is quite clear that some test of the above kind is absolutely needful in all biometric enquiries in which we wish to test theory against observation. In the paper referred to a small table for $P$ in terms of $n^{\prime}$ and $\chi^{3}$ was given, but this table beside being far from extensive enough for actual practice, was based in some entries on values of the probability integral which had not been calculated by the use of higher differences. The present Table $I$ is an attempt to provide a more extensive and accurate system of values for $P$. It gives the values of $P$ for $n^{\prime}=3$ to 30 and from $\chi^{2}=1$ to 30 by units and from $x^{3}=30$ to 70 by tens.

\section{Method of Calculating Tables.}

In order to simplify the work of calculating $P$ for values lying outside the range of this table, or in cases where interpolation would not give sufficiently accurate results a series of additional tables are given which were used in the calculation of Table I. Thus Table II. gives the values of $\log \left(x \sqrt{\frac{\varepsilon}{\pi}} e^{-i x}\right)$ and $\log \left(\sigma^{\left.-1 x^{*}\right)}\right.$ to eight figures Table V. gives $\log \sigma^{-1}$ and $\log \sqrt{\frac{2}{\pi}}$ to ten figares*. Table III. gives the cologarithms of $n(n-2)(n-4) \ldots 1$ (or 2) needed for the coefficients of the powers of $\chi$ to eight figures. Table IV. gives the values of $\sqrt{\frac{2}{\pi}} \int_{x}^{\infty} \sigma-i x^{3} d \chi$ for $\chi^{3}=1$ to 30 to eight fgures, ie. as long as it is practically sensible. Further values of this integral may be deduced from the tables for $\frac{2}{\sqrt{\pi}} \int_{0}^{t} \sigma^{-n} d t$, which are given for $t=0$ to 4.80 to eleven places of decimals for the higher values in Czuber's Theoris der Beobachtungsfehler, Leipzig, 1891.

In calculating the tables Erskine Scott's 10-Figure Logarithms and Filipowski's 7-Figure Antilogarithms were used. The method of calculation was, briefly, as follows. Tables were first made of $\log \left(\sqrt{\frac{2}{\pi}} \sigma x^{2}\right)$ and $\log \sigma^{-i x}$ by continuous

- Thas incidentally the ordinstes of the normal probability curre, $y=1 \sqrt{\frac{8}{8}} e^{-t \infty}$, are given for the equares of the abeoleses. 
addition and after adding $\log \chi$ to the former, the resulting figures were reduced from ten to eight places of decimals 80 as to aroid the error that would arise from the accumulation of amall differences in the eleventh place in the value of $\log e^{-1}$. These tables were carefully checked by addition and by examining every tenth value in the continuous work. The table of colog $[n]\left(=\log \frac{1}{n(n-2)(n-4) \ldots}\right)$ was originally calculated to ten places. The only other auxiliary table required was for $\sqrt{\frac{2}{\pi}} \int_{x}^{\infty}{ }^{-b x^{2}} d x$, and these values were calculated to seven places of decimals by second differences from a table of values of $\frac{2}{\sqrt{\pi}} \int_{0}^{t} \sigma^{-*} d t^{*}$. It was quite safe to omit third differences. The values of $P$ were then calculated from formulm (i) and (ii) given above. In making the table, to find $\chi^{m}$, a column of $s \log \chi^{2}$ was first set up, and then by means of four moveable slips of paper (two for $n^{\prime}$ even and two for $n^{\prime}$ odd) a second column calculated giving the sum of $8 \log \chi^{2}, \operatorname{colog}(2 s+1)$ and $\log \left(\sqrt{\frac{2}{\pi}} \chi^{-t+x^{2}}\right)$. These figures were checked by addition. The use of slips with colog $(n)$ written on them saved a very large amount of copying. The antilogarithms of the items of the second column were then put in a third column and the values of $\sqrt{\frac{2}{\pi}} \int_{x}^{\infty} \sigma-1 x^{2} d x$ having been written at the top of it, the figures given in Table I. were found by continuous addition. The values for $n^{\prime}$ even were calculated in like manner. The numbers obtained were tested when possible against those originally published in the Phil. Mag. and against a few additional values calculated by Miss M. A. Lewenz. The work was of course checked at every stage, but when the table was completed the second differences in each column were examined and found to run smoothly. The like method of differences was appealed to in the case of discrepancies between the short table and the present table, which were not due to the approximate value taken for the probability integral. It is hoped that the table as it now stands is substantially free from error.

In using the present method of testing goodness of fit it is essential to bear in mind a warning given in the paper in the Phil. Mag. referred to above (footnote, p. 164): "A theoretical probability curve without limited range will never at the extreme tails exactly fit observation. The difficulty is obvious where the observations go by units and the theory by fractions. We ought to take our final theoretical groups to cover as much of the tail area as amounts to at least a unit of frequency in such cases"

Further we ought to be careful to read the corresponding areas of the frequency curve and not merely the mid-ordinates, when we have not a great number of groups, or when, although the groups are numerous, the frequency is very skew.

- The Table in Galloweg's Treative on Probabilitice was the one actallly ared. 


\section{Illustration of woo of Tables.}

In the table below we have the distribution of the cephalic index in 900 skulls of modern Bavarian peasants. The frequency is given in the second column. In the third column we have the distribution as indicated by the normal curve of errors. Is this a reasonable description of the series of measurements ? In the fourth column are given the values of $m_{r}-m_{r}$ and in the fifth those of $\left(m_{r}-m_{r}\right)^{2} / m_{r}$. The resulting value of $\chi^{2}$ is 18.36 and $n^{\prime} \infty 24$. Table I. gives us: $n^{\prime}=24, \chi^{2}=18, P=757489$, and $\chi^{2}=19, P=701224$. Hence the required probability is nearly 737 , or roughly in every three cases out of four a random sampling would lead to a system of deviations diverging more widely from theory. Thus the fit may be considered a good one.

Cephalio Index of Bavarian Skulls.

\begin{tabular}{|c|c|c|c|c|}
\hline Index & Obserred & Clouleted & $m_{\mathbf{r}}-\boldsymbol{m}_{\mathbf{p}}$ & $\frac{\left(m_{r}-m_{r}\right)^{2}+}{m_{r}}$ \\
\hline $\begin{array}{c}\text { Under } 71 \cdot 5 \\
78 \\
73 \\
74 \\
75 \\
76 \\
77 \\
78 \\
79 \\
80 \\
81 \\
88 \\
83 \\
84 \\
85 \\
86 \\
87 \\
88 \\
89 \\
80 \\
91 \\
88 \\
83 \\
\text { Over } 93 \cdot 5\end{array}$ & $\begin{array}{l}8 \\
0 \\
8 \cdot 5 \\
1 \cdot 5 \\
3 \cdot 5 \\
12 \cdot 5 \\
17 \\
37 \\
55 \\
71 \cdot 5 \\
82 \\
116 \\
88 \\
107 \\
88 \\
74 \\
58 \\
34 \cdot 5 \\
19 \\
10 \\
8 \\
3 \\
1 \cdot 5 \\
4 \cdot 5\end{array}$ & $\begin{array}{l}1 \\
1 \\
1 \cdot 5 \\
3 \cdot 5 \\
7 \cdot 5 \\
13 \cdot 5 \\
23 \\
35 \cdot 5 \\
58 \cdot 5 \\
69 \cdot 5 \\
86 \\
98 \cdot 5 \\
103 \\
99 \cdot 5 \\
88 \cdot 5 \\
78 \\
54 \\
37 \cdot 5 \\
23 \cdot 5 \\
14 \\
7 \cdot 5 \\
3 \cdot 5 \\
2 \\
8\end{array}$ & $\begin{array}{l}-1 \\
+1 \\
-1 \\
+8 \\
+4 \\
+1 \\
+6 \\
-1.5 \\
-8.5 \\
-8 \\
+4 \\
-17.5 \\
+5 \\
-7.5 \\
+6.5 \\
-8 \\
-4 \\
+3 \\
+4.5 \\
+4 \\
-0.5 \\
+0.5 \\
+0.5 \\
-8.5\end{array}$ & $\begin{array}{l}1 \\
1 \\
67 \\
1 \cdot 14 \\
2 \cdot 13 \\
07 \\
1 \cdot 57 \\
08 \\
\cdot 18 \\
08 \\
\cdot 19 \\
8 \cdot 11 \\
24 \\
\cdot 57 \\
\cdot 48 \\
06 \\
\cdot 30 \\
24 \\
\cdot 86 \\
1 \cdot 14 \\
03 \\
07 \\
\cdot 126 \\
3 \cdot 126\end{array}$ \\
\hline Totals & 800 & 800 & 0 & $x^{3}-18 \cdot 36$ \\
\hline
\end{tabular}

\footnotetext{
- The aldoulated values are given to the nearest hall stull because the observed velues only run to this anit.

+ The numbers in the 6 fth colamn were obtained from the equares of those in the fourth by dividing them by the correuponding numbers in the third. The squaring is at once done from Barlow's Tables and the division to the scooracy required by Crelle's Rechentafeln. Both these books are indispensable to biometrioient.
} 
TABLE I.

\begin{tabular}{|c|c|c|c|c|c|c|c|c|c|}
\hline$x^{2}$ & $x^{\prime}=8$ & $x^{\prime}=4$ & $n^{\prime}=5$ & $n^{\prime}=6$ & $x^{\prime}=7$ & $x^{\prime} x 8$ & $n^{\prime}=9$ & $n^{\prime}=10$ & $n^{\prime}=11$ \\
\hline 1 & 606531 & 801253 & 809788 & 962566 & 85612 & $\theta 94829$ & $99824 \theta$ & 999438 & 899828 \\
\hline 2 & $\cdot \mathbf{9 6 7 8 7 \theta}$ & $\cdot 678407$ & 735759 & 849146 & 919099 & 968840 & 881018 & 991468 & -896840 \\
\hline$s$ & -223130 & 391625 & 557826 & 690986 & 808847 & 886008 & -934367 & 964896 & 981484 \\
\hline 4 & $\cdot 135335$ & -201464 & - 406000 & .549416 & 676676 & 779778 & 857123 & 011418 & -947347 \\
\hline 5 & 082086 & $\cdot 171797$ & 287898 & 415880 & ·543813 & 669063 & .57576 & 834308 & $\$ 1178$ \\
\hline 6 & 049787 & & $\cdot 199148$ & 306219 & .483180 & .539750 & 847899 & 7399918 & 815268 \\
\hline 7 & 030197 & 071897 & $\cdot 135888$ & 280640 & $\cdot 380847$ & -488880 & .5360092 & $\$ 37119$ & 725444 \\
\hline 8 & 018316 & 046012 & 001578 & $\cdot 156236$ & 238103 & 332594 & .439470 & $\cdot 534146$ & 628837 \\
\hline $\boldsymbol{g}$ & .011109 & 020201 & 081090 & $\cdot 109064$ & $\cdot 173578$ & 252656 & 349296 & $\cdot 437874$ & .538104 \\
\hline 10 & 006738 & 018666 & 040428 & 075235 & $\cdot 124652$ & $\cdot 188673$ & 265026 & 350485 & 440403 \\
\hline 11 & .004087 & 011728 & 026564 & 0.51380 & 088378 & $\cdot 138619$ & 201699 & -275709 & .357518 \\
\hline 12 & $00847 \theta$ & 007383 & 017351 & 034787 & 061869 & $\cdot 100568$ & .151804 & 213308 & 286057 \\
\hline 13 & 0016003 & 004637 & 011276 & 023379 & 043036 & 072109 & $\cdot 111850$ & -162607 & .223672 \\
\hline 14 & 000918 & 002005 & 0072095 & $015+009$ & 029636 & 0.51181 & 081765 & & 78998 \\
\hline 15 & 000653 & $\infty 01817$ & 004701 & 010383 & 020256 & 036000 & 059145 & 000937 & $\cdot 132001$ \\
\hline 16 & 000335 & $\infty 01134$ & 003019 & 006844 & 013754 & 025116 & 048380 & 066881 & 099638 \\
\hline 17 & 000203 & 000707 & 001833 & 004500 & 009283 & 017396 & 030109 & 048716 & 074364 \\
\hline 18 & 000193 & 000440 & 001234 & 002847 & 006832 & 011970 & 021226 & 035174 & 0.54864 \\
\hline 19 & 000075 & 000273 & 000786 & 001922 & 004164 & 008187 & 014860 & 3193 & 040889 \\
\hline 20 & 000046 & 000170 & 000489 & 001250 & 008769 & 005670 & 010338 & 017913 & 020253 \\
\hline 21 & 000028 & 000105 & 000317 & 000810 & 001835 & 003770 & 007147 & 018050 & 021003 \\
\hline 28 & 000017 & 000065 & 000200 & 000584 & 001211 & 002541 & 004916 & 008880 & 015100 \\
\hline 28 & 000010 & 000040 & 000187 & 000338 & .000786 & 001708 & -003364 & 006187 & 010747 \\
\hline 24 & 000006 & 000025 & 000080 & 000217 & 000528 & 001139 & 002898 & $\infty 04301$ & 007800 \\
\hline 25 & 000004 & $\infty 00016$ & 000050 & 000139 & 000341 & 000759 & 001554 & 002871 & 0,05346 \\
\hline 26 & 000008 & 000010 & 000038 & 000090 & 000283 & 000504 & 001050 & 008043 & 003740 \\
\hline 27 & 000001 & 000000 & 000020 & 000037 & 000145 & 000333 & 000707 & $\infty 0139 \theta$ & 002004 \\
\hline 28 & 000001 & 000004 & 000018 & 000037 & 000094 & 000280 & 000474 & 000864 & 001806 \\
\hline 29 & 000001 & $0000 \dot{0 z}$ & 000008 & 000023 & 000061 & 000145 & 000317 & 000648 & 001246 \\
\hline so & 000000 & -000001 & 00000 s & 000015 & 000039 & 000095 & 000211 & 000439 & 000857 \\
\hline 40 & .000000 & 000000 & 000000 & & 000001 & 000001 & 000003 & 0008 & 000017 \\
\hline 50 & 000000 & 000000 & 000000 & & 000000 & .000000 & 000000 & & 000000 \\
\hline 60 & 000000 & 000000 & 000000 & & 000000 & 000000 & 000000 & 000000 & 000000 \\
\hline 70 & 000000 & 000000 & 000000 & 000000 & 000000 & .000000 & 000000 & 000000 & 000000 \\
\hline
\end{tabular}


TABLE I.-continued

\begin{tabular}{|c|c|c|c|c|c|c|c|c|c|}
\hline$x^{3}$ & $n^{\prime}=12$ & $n^{\prime}=18$ & $x^{\prime}=14$ & $m^{\prime}=16$ & $n^{\prime}=16$ & $x^{\prime}=17$ & $x^{\prime}=18$ & $x^{\prime}=18$ & $x^{\prime}=20$ \\
\hline 1 & 999950 & 909986 & 999997 & 000999 & $1 \cdot$ & $1 \cdot$ & $1 \cdot$ & $1 \cdot$ & 1. \\
\hline $\boldsymbol{2}$ & 988496 & 990408 & 899774 & 909017 & 999970 & $\cdot 0000000$ & 999997 & 9899989 & $1 \cdot$ \\
\hline$s$ & 900786 & 905644 & 997834 & 999074 & -999588 & 990830 & 990931 & 999978 & 999989 \\
\hline 4 & 969917 & 983436 & 891191 & -996468 & 997737 & 9089003 & 909483 & 999763 & 099894 \\
\hline 5 & 031167 & $-05797 \theta$ & 975193 & 986813 & 992187 & 995754 & 907771 & 998860 & 090481 \\
\hline$\theta$ & 873365 & $\theta 16082$ & -046163 & 866491 & 979749 & 988095 & 993187 & 990197 & 907929 \\
\hline 7 & $\cdot 790073$ & -857613 & 002161 & 034711 & 957650 & 973800 & 083549 & 990125 & 894813 \\
\hline 8 & 713304 & 780131 & 843601 & 889397 & 993783 & 948867 & 866547 & 978637 & 986671 \\
\hline 9 & 621898 & 702931 & $\cdot 772943$ & .831051 & 877517 & -913414 & $\theta 40281$ & 959743 & 973479 \\
\hline 10 & .630387 & 615800 & 093934 & $\cdot 762183$ & $\cdot 819739$ & .866628 & 803610 & 231800 & -952946 \\
\hline 11 & $\cdot 443263$ & $\cdot 588819$ & 610817 & 686038 & 752594 & 809486 & 856564 & 894367 & 923839 \\
\hline 18 & 382649 & .445680 & $\cdot 687643$ & 606303 & 879028 & 743980 & $\cdot 800136$ & $\cdot 847237$ & $\cdot 885684$ \\
\hline 13 & 293328 & -360041 & $\cdot 447818$ & $\cdot 526524$ & 602898 & 672758 & $\cdot 738186$ & $\cdot 791673$ & .838571 \\
\hline 14 & .2329993 & $\cdot 300708$ & $\cdot 373844$ & $\cdot 449711$ & $\cdot 525589$ & $\cdot 598714$ & 6867102 & $\cdot 780091$ & $\cdot 783691$ \\
\hline 15 & $\cdot 182408$ & -841436 & 307354 & $\cdot 378154$ & $\cdot 461418$ & $\cdot 524638$ & 595482 & 681907 & 722588 \\
\hline 16 & $\cdot 141130$ & $\cdot 181236$ & 249129 & 313374 & 382051 & .452961 & $\cdot 523834$ & $\cdot 608547$ & .657877 \\
\hline 17 & $\cdot 107876$ & $\cdot 149507$ & $\cdot 180304$ & 206178 & 318864 & 386597 & • 4543968 & .683105 & $\cdot 689868$ \\
\hline 18 & 081681 & $\cdot 115691$ & $\cdot 167580$ & 208781 & 268666 & $\cdot 323897$ & -388841 & $\cdot 4566653$ & .522438 \\
\hline 19 & 061004 & 088529 & $\cdot 123104$ & $\cdot 164949$ & -213734 & -268683 & $\cdot 388532$ & $\cdot 391883$ & $\cdot 456836$ \\
\hline 20 & 045341 & 087086 & 005210 & $\cdot 130141$ & $\cdot 171832$ & 220290 & -274899 & $\cdot 332819$ & $\cdot 394578$ \\
\hline e! & 033371 & 0.00380 & 072929 & $\cdot 101638$ & $\cdot 136830$ & $\cdot 178510$ & 226891 & -270413 & 336801 \\
\hline 28 & 084374 & 037590 & 0.55368 & 078614 & $\cdot 107804$ & $\cdot 143191$ & $\cdot 18471 \theta$ & 231886 & 284256 \\
\hline 23 & 017676 & 027728 & 041677 & 060270 & 084140 & $\cdot 113735$ & $\cdot 149251$ & $\cdot 190680$ & .237348 \\
\hline 24 & 018733 & 020341 & -031130 & 045882 & 085023 & 089504 & $\cdot 119435$ & $\cdot 156028$ & $\cdot 196158$ \\
\hline 25 & 009117 & 014892 & 023084 & 034566 & 049843 & 069884 & 024710 & $\cdot 124815$ & $\cdot 160549$ \\
\hline 26 & 000490 & 010734 & 017001 & 025887 & 038023 & 054028 & 074461 & 099738 & $\cdot 13018 \theta$ \\
\hline 87 & 004595 & 007727 & 012441 & 019254 & 028736 & 041483 & 0.58068 & 078996 & $\cdot 104653$ \\
\hline 28 & 003238 & 005538 & 009050 & 014928 & 021060 & 031620 & 044938 & 069056 & 083488 \\
\hline 29 & 002870 & 003940 & 000546 & 010460 & 016085 & 023936 & 034586 & 048379 & 085985 \\
\hline 30 & 001585 & 002792 & 004710 & 007632 & 011981 & 018002 & 020345 & 037446 & 0.51798 \\
\hline 40 & -000036 & 000078 & 000138 & -000255 & 000463 & 000778 & 001894 & 002087 & 003278 \\
\hline 50 & 000001 & 000001 & 000003 & 000006 & 000012 & 000023 & 000042 & -000075 & 000131 \\
\hline 60 & 000000 & 000000 & 000000 & 000000 & 000000 & -000001 & 000001 & 000002 & 000004 \\
\hline 70 & 000000 & 000000 & 000000 & 000000 & 000000 & 000000 & 000000 & 000000 & 000000 \\
\hline
\end{tabular}


TABLE I.-continued.

\begin{tabular}{|c|c|c|c|c|c|c|c|c|c|c|}
\hline$x^{\mathbf{a}}$ & $x^{\prime}=91$ & $x^{\prime}=88$ & $x^{\prime}=88$ & $n^{\prime}=24$ & $n^{\prime}=25$ & $x^{\prime}=28$ & $x^{\prime}=87$ & $n^{\prime}=28$ & $x^{\prime}=29$ & $x^{\prime}=80$ \\
\hline 1 & $1 \cdot$ & 1. & I. & 1 . & 1. & 1. & $1^{\circ}$ & 1 . & 1. & $1 \cdot$ \\
\hline 2 & 1. & 1. & 1. & 1. & 1. & 1 . & 1. & 1. & $1^{\circ}$ & 1. \\
\hline$s$ & 998096 & .999008 & 999990 & 1. & 1. & 1. & 1 . & 1. & $1 \cdot$ & 1. \\
\hline 4 & 899954 & 999890 & 999998 & 990997 & 989999 & $1 \cdot$ & 1. & 1. & 1. & 1. \\
\hline 5 & 999729 & 998868 & 989939 & 299978 & 299887 & 990904 & 999998 & 989999 & 1. & 1. \\
\hline 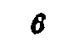 & 998808 & $989<87$ & 999708 & 989856 & 999929 & 900068 & 999084 & 999998 & 909897 & $998009 \theta$ \\
\hline 7 & 906888 & 898148 & 998890 & 090468 & 999711 & 909851 & 990984 & 809009 & 890081 & 900091 \\
\hline 8 & $9 \theta 1868$ & 995143 & 997160 & 988371 & 909086 & 800104 & 60.9728 & 298953 & 899924 & 800000 \\
\hline 9 & 288907 & 980214 & 993331 & 895957 & 097005 & 988580 & 899184 & 999546 & 899748 & 999863 \\
\hline 10 & 868171 & 978912 & 886504 & 991877 & 804647 & 800053 & 997881 & -998803 & 900302 & 999599 \\
\hline 11 & +46898 & 868787 & $97474 \theta$ & 283169 & 880012 & 902048 & 995040 & 997839 & 008316 & 998988 \\
\hline 18 & $\theta 16076$ & 830617 & 967379 & $971 \times 70$ & -970908 & 886567 & 991173 & 904894 & 896379 & 997798 \\
\hline 19 & $\cdot 877384$ & 908684 & 933161 & 251990 & 968191 & 976501 & 983974 & 289847 & 002000 & 895381 \\
\hline 14 & 830406 & 868598 & 801479 & 928871 & 046660 & 961738 & 873000 & 081254 & 887189 & 991377 \\
\hline 15 & $\cdot 776408$ & 889968 & 868938 & 894634 & 920759 & 941383 & 957334 & .969438 & 978436 & 986016 \\
\hline 16 & 716894 & 769660 & 816886 & 855968 & 888076 & 914828 & 936203 & 258947 & 805819 & 875536 \\
\hline 17 & 658074 & 711106 & 763368 & 809251 & 848668 & 881703 & 800083 & 231188 & 248589 & 908181 \\
\hline 18 & .587408 & 648004 & 705988 & 757489 &.-803008 & 8498300 & 875773 & $80381 \theta$ & $-\theta z \theta 14 \theta$ & 944872 \\
\hline 19 & .521828 & 585140 & 645328 & 701824 & 751990 & 797180 & 836430 & 870001 & 898136 & 981288 \\
\hline 20 & $\cdot 457830$ & .021281 & .583040 & 841012 & 698776 & 746825 & $\cdot 791556$ & 830756 & 864464 & 898927 \\
\hline 21 & 397132 & 458941 & $\cdot 680738$ & .681087 & $\$ 38725$ & 692609 & 741864 & 786988 & 826349 & -859149 \\
\hline 28 & 340511 & 399510 & -458889 & .520259 & .579807 & 636744 & 688697 & 737377 & $\cdot 781891$ & 890189 \\
\hline 25 & 288795 & 343979 & -401730 & $\cdot 460771$ & .519788 & .577564 & 632947 & 880013 & $\cdot 733041$ & 776643 \\
\hline 24 & 248398 & 283058 & 347828 & $\cdot 403808$ & $\cdot 461597$ & 518373 & .575065 & 680316 & 681535 & $\cdot 728838$ \\
\hline 25 & 201431 & $\cdot 247164$ & 297075 & .360285 & $\cdot 40.5760$ & $\cdot 462873$ & .518975 & .874468 & 687835 & 878248 \\
\hline 26 & -165818 & 200440 & 251682 & $\cdot 300868$ & $\cdot 353165$ & $\cdot 407588$ & $\cdot 403105$ & .518600 & .573046 & 625491 \\
\hline 27 & $\cdot 135284$ & $\cdot 170853$ & 211220 & 2655807 & 301453 & 355884 & $\cdot 409333$ & $\cdot 463794$ & $\cdot 518247$ & .571705 \\
\hline 28 & '108399 & - 140151 & $\cdot 175681$ & -815781 & 260040 & 307853 & $\cdot 358458$ & $\cdot 410973$ & $\cdot 464447$ & .517913 \\
\hline $2 \theta$ & 087769 & -114002 & $\cdot 144861$ & - 180310 & 280131 & 263910 & .311088 & 360899 & -412328 & $\cdot 465068$ \\
\hline so & 0698854 & 091888 & $\cdot 118464$ & - 149402 & $\cdot 184768$ & 224280 & 267811 & 314154 & .383818 & .414004 \\
\hline 40 & 004995 & .007437 & .010818 & 015360 & 021387 & 029164 & 039018 & 051237 & 066128 & 083937 \\
\hline 50 & 000221 & 000365 & 000586 & 000921 & $\infty 01416$ & 002131 & 003144 & DC45s & 000467 & .009032 \\
\hline 60 & 000007 & 000013 & 000028 & 000038 & 000004 & 000104 & 000168 & 000284 & 000407 & 000618 \\
\hline 70 & 000000 & 000000 & 000001 & 000001 & 000002 & 000004 & 000007 & $-\infty 00011$ & 000018 & 000030 \\
\hline
\end{tabular}


TABLE II

\begin{tabular}{|c|c|c|c|c|c|}
\hline$x^{3}$ & $\log \left\{x \sqrt{\frac{2}{\pi}} e^{-b x^{8}}\right.$ & $\log e^{-1}+x^{4}$ & $x^{2}$ & $\log \left\{x \sqrt{\frac{8}{x}} e^{-b}\right.$ & $\log _{0}-1 x^{2}$ \\
\hline $\begin{array}{l}1 \\
8 \\
3 \\
4 \\
5 \\
6 \\
7 \\
8 \\
9 \\
10 \\
11 \\
18 \\
13 \\
14 \\
15 \\
16 \\
17 \\
18 \\
19 \\
80 \\
21 \\
28 \\
23 \\
24 \\
25 \\
26 \\
87 \\
28 \\
29 \\
\$ 0 \\
\$ 1 \\
\$ 2 \\
\$ 9 \\
\$ 4 \\
35 \\
36 \\
57 \\
\$ 8 \\
\$ 9 \\
40 \\
41 \\
48 \\
45 \\
44 \\
45 \\
46 \\
47 \\
48 \\
49 \\
50\end{array}$ & 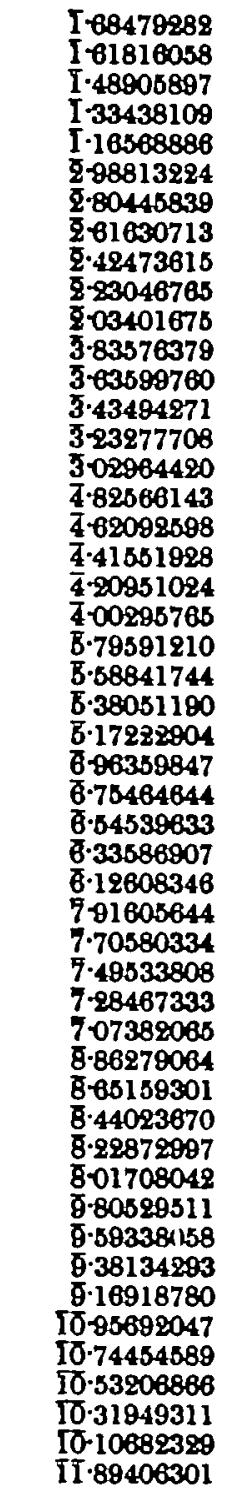 & 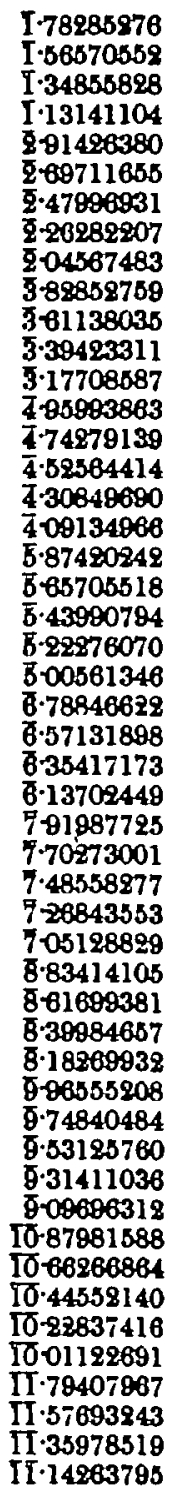 & $\begin{array}{c}51 \\
58 \\
59 \\
54 \\
55 \\
56 \\
57 \\
68 \\
59 \\
60 \\
61 \\
68 \\
69 \\
64 \\
65 \\
68 \\
67 \\
68 \\
69 \\
70 \\
71 \\
78 \\
73 \\
74 \\
75 \\
76 \\
77 \\
78 \\
79 \\
80 \\
81 \\
88 \\
89 \\
84 \\
85 \\
86 \\
87 \\
88 \\
89 \\
90 \\
91 \\
98 \\
98 \\
94 \\
95 \\
96 \\
97 \\
98 \\
99 \\
100\end{array}$ & 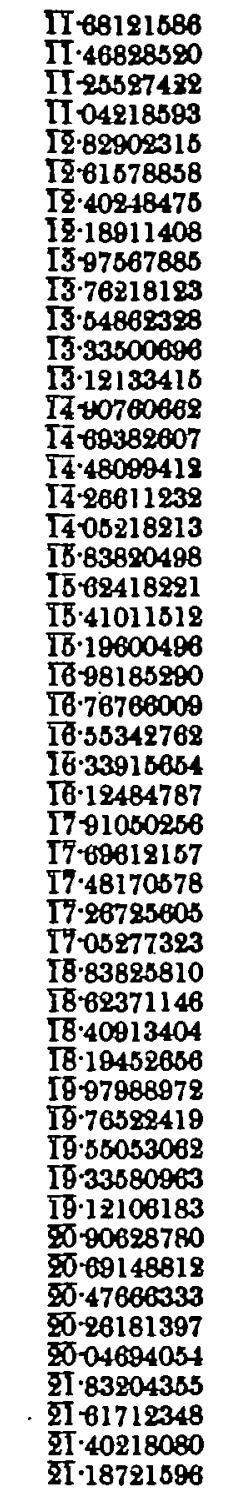 & 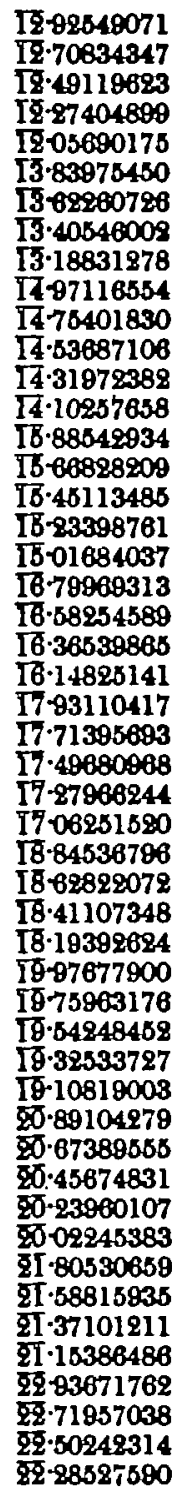 \\
\hline
\end{tabular}


TABLE III.

Table of colog $[n]:-[n]=n(n-2)(n-4) \ldots \ldots$

\begin{tabular}{|c|c|c|c|}
\hline odd noe. & $\infty \log [x]$ & epen nos. & $\infty 0 \log [R]$ \\
\hline 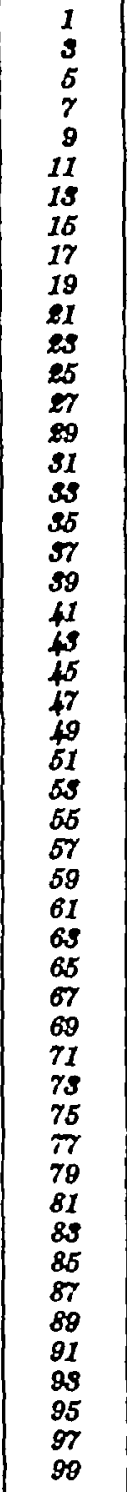 & 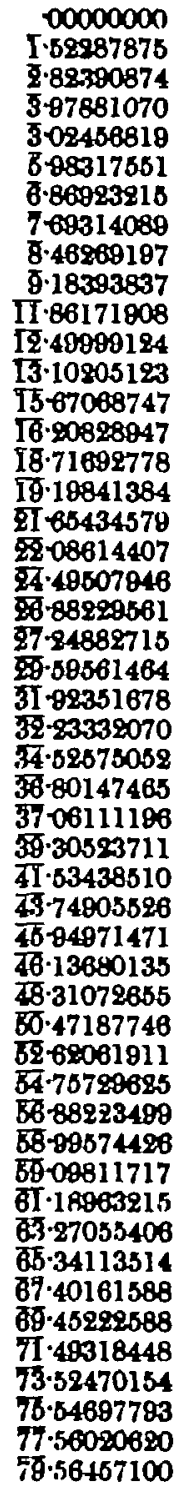 & $\begin{array}{l}2 \\
4 \\
6 \\
8 \\
10 \\
12 \\
14 \\
16 \\
18 \\
80 \\
28 \\
24 \\
26 \\
28 \\
50 \\
38 \\
54 \\
56 \\
38 \\
40 \\
42 \\
44 \\
46 \\
48 \\
50 \\
52 \\
54 \\
56 \\
58 \\
60 \\
62 \\
64 \\
66 \\
68 \\
70 \\
78 \\
74 \\
76 \\
78 \\
80 \\
82 \\
84 \\
86 \\
88 \\
30 \\
98 \\
94 \\
96 \\
98 \\
100\end{array}$ & 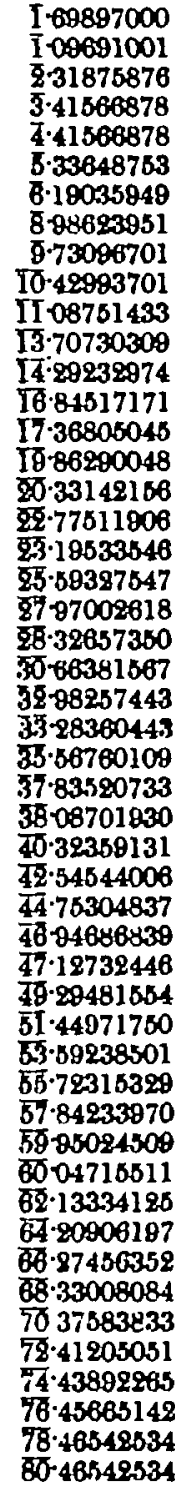 \\
\hline
\end{tabular}

TABLE IV.

\begin{tabular}{|c|c|}
\hline$x^{2}$ & $\frac{\overline{2}}{\bar{x}} \int_{x}^{\infty} e^{-1}$ \\
\hline $\begin{array}{l}1 \\
2 \\
5 \\
4 \\
5 \\
6 \\
7 \\
8 \\
9 \\
10 \\
11 \\
12 \\
13 \\
14 \\
15 \\
16 \\
17 \\
18 \\
19 \\
20 \\
21 \\
22 \\
25 \\
24 \\
25 \\
26 \\
27 \\
28 \\
29 \\
50\end{array}$ & $\begin{array}{l}\cdot 8173106 \\
1672998 \\
0832446 \\
0455003 \\
00263474 \\
0143000 \\
0081506 \\
0046776 \\
0026908 \\
0015654 \\
0009118 \\
0005321 \\
0003115 \\
0001828 \\
0001076 \\
0000634 \\
0000374 \\
0000891 \\
0000138 \\
0000078 \\
0000046 \\
0000027 \\
0000016 \\
0000011 \\
0000007 \\
0000004 \\
0000003 \\
0000008 \\
0000001 \\
0000000\end{array}$ \\
\hline
\end{tabular}

TABLE $\nabla$.

\begin{tabular}{|c|c|}
\hline Punotion & Lob. Funetion \\
\hline $0-1$ & $I \cdot 7828527580$ \\
\hline$\sqrt{\frac{2}{\pi}}$ & I 8018400815 \\
\hline
\end{tabular}

